\title{
Spiking Neural P Systems with Structural Plasticity
}

\author{
Francis George C. Cabarle ${ }^{1}$, Henry N. Adorna ${ }^{1}$, Mario J. Pérez-Jiménez ${ }^{2}$, Tao \\ Song 3 \\ ${ }^{1}$ Algorithms \& Complexity Lab, Department of Computer Science \\ University of the Philippines Diliman \\ Diliman 1101 Quezon City, Philippines; \\ ${ }^{2}$ Department of Computer Science and AI \\ University of Sevilla \\ Avda. Reina Mercedes s/n, 41012, Sevilla, Spain; \\ ${ }^{3}$ School of Automation \\ Huazhong University of Science and Technology \\ Wuhan 430074, Hubei, China \\ email: fccabarle@up.edu.ph, hnadorna@dcs.upd.edu.ph, marper@us.es, \\ taosong@hust.edu.cn
}

\begin{abstract}
Spiking neural P (SNP) systems are a class of parallel, distributed, and nondeterministic computing models inspired by the spiking of biological neurons. In this work, the biological feature known as structural plasticity is introduced in the framework of SNP systems. Structural plasticity refers to synapse creation and deletion, thus changing the synapse graph. The "programming" therefore of a brain-like model, the SNP system with structural plasticity (SNPSP system), is based on how neurons connect to each other. SNPSP systems are also a partial answer to an open question on SNP systems with dynamism only for synapses. For both the accepting and generative modes, we prove that SNPSP systems are universal. Modifying SNPSP systems semantics, we introduce the spike saving mode and prove that universality is maintained. In saving mode however, a deadlock state can arise, and we prove that reaching such a state is undecidable. Lastly, we provide one technique in order to use structural plasticity to solve a hard problem: a constant time, nondeterministic, and semi-uniform solution to the NP-complete problem Subset Sum.
\end{abstract}

Key words: Membrane computing, Spiking neural P systems, Structural plasticity, Computational universality, Deadlock, Undecidability, Subset sum

\section{Introduction}

Membrane computing is a branch of natural computing, aiming to abstract and obtain ideas (e.g. data structures, control operations, models) from the structure 
and functioning of biological cells [18. The idea of spiking in biological neurons have been introduced in the framework of membrane computing as spiking neural P systems (SNP systems in short) 9. In spiking neurons and in SNP systems, the indistinct signals known as spikes do not encode the information. Instead, information is derived from the time difference between two spikes, or the number of spikes sent (received) during the computation. Time therefore is an information support in spiking neurons, and not simply a background for performing computations.

An SNP system can be thought of as a network of spike processors, processing spike objects and sending them to other neurons. Essentially, SNP systems can be represented by directed graphs where nodes are neurons (often drawn as ovals) and edges between neurons are synapses. Spikes (represented as a multiset of the symbol $a$ ) are sent from one neuron to another using their synapses. In SNP system literatures, many biologically inspired features have been introduced for computing use, producing many SNP system variants: see e.g. [5] 17 [16 17] [20] 22] 24] 25] 27] and references therein.

In this work we are interested in the biological feature known as neural plasticity which is concerned with synapse modifications. Related to this feature are works in SNP systems with some forms of neural plasticity: Hebbian SNP (HSNP) systems 17 and SNP systems with neuron division and budding $[16$ 27. In HSNP systems, given two neurons $\sigma_{i}$ and $\sigma_{j}$, and a synapse $(i, j)$ between them, if spikes from neuron $\sigma_{i}$ arrive repeatedly and shortly before neuron $\sigma_{j}$ sends its own spikes, the synapse weight (strength) of $(i, j)$ increases. If however the spikes from neuron $\sigma_{i}$ arrive after the spikes of $\sigma_{j}$ are sent, synapse weight of $(i, j)$ decreases. HSNP systems were introduced for possible machine learning use in the framework of SNP systems.

In 16 27, other than spiking rules (rules that allow a neuron to send spike to other neurons), two new rules are introduced: neuron division and neuron budding rules. Both new rules involve creation of novel synapses (synaptogenesis) due to creation of novel neurons (neurogenesis). The initial synapse graph of the system is thus changed due to the application of the new rules, creating exponential workspace (in terms of neurons) in linear time. The SAT problem was then efficiently solved in 16 27, but by using the exponential workspace created.

The particular type of neural plasticity we introduce in the framework of SNP systems in this work is structural plasticity. Structural plasticity is concerned with any change in connectivity between neurons, with two mechanisms: (1) synaptogenesis and synapse deletion, (2) synaptic rewiring 2. Unlike Hebbian (also known as functional) plasticity which concerns itself with only two neurons (for synapse strength modification), synaptic rewiring involves at least three neurons: if we have three neurons $\sigma_{i}, \sigma_{j}, \sigma_{k}$ and only one synapse $(i, j)$, in order to create a synapse $(i, k)$ then synapse $(i, j)$ must be deleted first ${ }^{1}$. Synaptogenesis is present during neuron division and budding due to neurogenesis, while synapse

1 This is inspired by synaptic homeostasis in biological neurons, where total synapse number in the system is left unchanged [2]. 
rewiring is not included, and synapse deletion is implied. In 16 27 a linear number of neurons forms the initial synapse graph of the SNP system. From the initial synapse graph, an exponential number of neurons can be created after some linear amount of time.

Biological neurons in adult human brains can reach more than billions in numbers, and each neuron can wire to thousands of other neurons. This phenomenon is another biological motivation in this work. In this work we can have a collection of (possibly exponential number of) neurons. The initial synapse graph can still be composed of a linear number of neurons wired or connected using synapses. However, we are only concerned with the creation and deletion of synapses over this collection for computing use. The synapse graph will then change: it is possible the system will connect an exponential number of neurons together at certain time steps (due to synapse creation) and make use of additional workspace; at other time steps the system can connect a linear or polynomial number of neurons (due to synapse deletion) and "release" additional workspace (in terms of neurons or synapses) from the system which are no longer needed. The introduction of spiking neural $\mathrm{P}$ systems with structural plasticity (SNPSP systems for short) is also a partial response to the open problem $\mathbf{D}$ in [22] where "dynamism" only for synapses is to be considered.

Furthermore, the standard SNP system originally from 9] included neurons with spiking rules that can have complex regular expressions, delays (in applying rules), and forgetting rules (rules that remove spikes from the system). A series of papers which proved computational universality (or simply universality, if there is no confusion) while simplifying the regular expressions of rules, removing delays or forgetting rules followed, e.g. 6 [6], with the most recent being [15]. In 8 for example, SNP systems can be universal with the following being true: rules are without delays; without using forgetting rules; regular expressions are simple. In 66 it was shown that universality is achieved with more restrictions: without delays and forgetting rules; without delays and simple regular expressions. In order to maintain universality while further simplifying the system, we look for using other biological motivations.

In 17, universality in SNP systems was achieved with the following features: rules are without delays, and all neurons only have exactly one and the same simple rule. The way to control or "program" the SNP systems in 17 was using additional structures from neuroscience called astrocytes. Astrocytes introduce nondeterminism in the system, allowing the system to have simple neurons (that is, neurons in 17 contain only the rule $a \rightarrow a$ ).

In this work we use the biological feature of structural plasticity to achieve universality with the following restrictions: (a) only a "small" number of neurons have plasticity rules (details to follow shortly), (b) neurons without plasticity rules are simple (they only contain the rule $a \rightarrow a$ ), and (c) without the use of delays and forgetting rules. We do not include additional neuroscience structures other than neurons and their synapses. The introduction of the structural plasticity feature, in order to "program" the system, allows the system to maintain universality even with restrictions (a) to (c). Note that the idea of programming 
how a network of neurons connect in order to perform tasks is an old one in computer science, see for example [26].

We then modify the semantics of SNPSP systems, introducing a spike saving mode. For SNPSP systems operating in such a mode, we prove that their computing power is not diminished. However, an interesting property called a deadlock state, can occur in an SNPSP system in saving mode. We then prove that reaching such a state in saving mode is undecidable for an arbitrary SNPSP system. Lastly, we provide a constant time solution to the numerical NP-complete problem Subset Sum, using only nondeterminism in synapses (using plasticity rules), and without still using forgetting rules and delays. The solution is semi-uniform, and not surprisingly, can require an exponential number of neurons. Our preliminary solution provides one possibility of using synaptic plasticity for solving hard problems.

This work is organized in the usual way as follows: preliminaries used in the rest of this work are given in Section 2 . Section 3 introduces the syntax and semantics of SNPSP systems, and an example is given in Section 4, universality of SNPSP systems is given in Section 5, a modification of the semantics of SNPSP systems, the spike saving mode, is introduced in Section 6, including the deadlock property and its undecidability; a solution to Subset Sum is given in Section 7 a brief summary followed by final discussions and further research interests are given in Section 8 .

\section{Preliminaries}

It is assumed that the readers are familiar with the basics of membrane computing (a good introduction is [19] with recent results and information in the $\mathrm{P}$ systems webpag $\AA^{2}$ and a recent handbook 23 ) and formal language theory (available in many monographs). We only briefly mention notions and notations which will be useful throughout the paper.

We denote the set of natural (counting) numbers as $\mathbb{N}=\{1,2, \ldots\}$. Let $V$ be an alphabet, $V^{*}$ is the set of all finite strings over $V$ with respect to concatenation and the identity element $\lambda$ (the empty string). The set of all non-empty strings over $V$ is denoted as $V^{+}$so $V^{+}=V^{*}-\{\lambda\}$. We call $V$ a singleton if $V=\{a\}$ and simply write $a^{*}$ and $a^{+}$instead of $\{a\}^{*}$ and $\{a\}^{+}$. If $a$ is a symbol in $V$, we write $a^{0}=\lambda$. A regular expression over an alphabet $V$ is constructed starting from $\lambda$ and the symbols of $V$ using the operations union, concatenation, and + . Specifically, $(i) \lambda$ and each $a \in V$ are regular expressions, (ii) if $E_{1}$ and $E_{2}$ are regular expressions over $V$ then $\left(E_{1} \cup E_{2}\right), E_{1} E_{2}$, and $E_{1}^{+}$are regular expressions over $V$, and (iii) nothing else is a regular expression over $V$. With each expression $E$ we associate a language $L(E)$ defined in the following way: (i) $L(\lambda)=\{\lambda\}$ and $L(a)=\{a\}$ for all $a \in V$, (ii) $L\left(E_{1} \cup E_{2}\right)=$ $L\left(E_{1}\right) \cup L\left(E_{2}\right), L\left(E_{1} E_{2}\right)=L\left(E_{1}\right) L\left(E_{2}\right)$, and $L\left(E_{1}^{+}\right)=L\left(E_{1}\right)^{+}$, for all regular expressions $E_{1}, E_{2}$ over $V$. Unnecessary parentheses are omitted when writing regular expressions, and $E^{+} \cup\{\lambda\}$ is written as $E^{*}$.

\footnotetext{
2 http://ppage.psystems.eu/
} 
By $N R E$ we denote the family of Turing computable sets of numbers, that is, $N R E$ is the family of length sets of recursively enumerable languages recognized by Turing machines. In proving computational universality, we use the notion of register machines. A register machine is a construct $M=\left(m, I, l_{0}, l_{h}, R\right)$, where $m$ is the number of registers, $I$ is the set of instruction labels, $l_{0}$ is the start label, $l_{h}$ is the halt label, and $R$ is the set of instructions. Every label $l_{i} \in I$ uniquely labels only one instruction in $R$. Register machine instructions have the following forms:

$-l_{i}:\left(\operatorname{ADD}(r), l_{j}, l_{k}\right)$, where the value in register $r$ is increased by 1 then nondeterministically go to either instruction label $l_{j}$ or $l_{k}$;

$-l_{i}:\left(\operatorname{SUB}(r), l_{j}, l_{k}\right)$, where if the value in register $r$ is nonzero, then subtract 1 from it and go to instruction label $l_{j}$, otherwise go to instruction label $l_{k}$;

$-l_{h}:$ HALT, the halt instruction.

Given a register machine $M$, we say $M$ computes or generates a number $n$ as follows: $M$ starts with all its registers empty. The register machine then applies its instructions starting with the instruction labeled $l_{0}$. Without loss of generality, we assume that $l_{0}$ labels an ADD instruction, and that the content of the output register is never decremented, only added to during computation, i.e. no SUB instruction is applied to it. If $M$ reaches the halt instruction $l_{h}$, then the number $n$ stored during this time in the first register is said to be computed by $M$. We denote the set of all numbers computed by $M$ as $N(M)$. It was proven that register machines compute all sets of number computed by a Turing machine, therefore characterizing NRE 13 .

Register machines can also work in an accepting mode. A number $n$ is stored in the first register of register machine $M$, with all other registers being empty. If the computation of $M$ starting with this initial configuration halts, then $n$ is said to be accepted or computed by $M$. In the accepting mode and even with $M$ being deterministic, i.e. given an $\mathrm{ADD}$ instruction $l_{i}:\left(\operatorname{ADD}(r), l_{j}, l_{k}\right)$ with $l_{j}=l_{k}$ written simply as $l_{i}:\left(\operatorname{ADD}(r), l_{j}\right)$, register machine $M$ can still characterize $N R E$.

As a convention in membrane computing, when comparing the power of two number generating or accepting devices $D_{1}$ and $D_{2}$, the number zero is ignored, i.e. $N\left(D_{1}\right)=N\left(D_{2}\right)$ if and only if $N\left(D_{1}\right)-\{0\}=N\left(D_{2}\right)-\{0\}$. This convention corresponds to the common practice in language and automata theory to ignore the empty string.

\section{Spiking Neural P Systems with Structural Plasticity}

In this section we introduce the variant of SNP systems with structural plasticity. The reader is invited to consult with the original SNP systems paper in [9] for initial motivations and preliminary results.

Formally, a spiking neural P system with structural plasticity (SNPSP system) of degree $m \geq 1$ is a construct of the form $\Pi=\left(O, \sigma_{1}, \ldots, \sigma_{m}\right.$, syn, in, out $)$, where: 
- $O=\{a\}$ is the singleton alphabet ( $a$ is called spike)

- $\sigma_{1}, \ldots, \sigma_{m}$ are neurons of the form $\left(n_{i}, R_{i}\right), 1 \leq i \leq m$, with $n_{i} \geq 0$ indicating the initial number of spikes in $\sigma_{i}$, and $R_{i}$ is a finite rule set of $\sigma_{i}$ with the following forms:

1. Spiking rule: $E / a^{c} \rightarrow a$, where $E$ is a regular expression over $O$, with $c \geq 1$

2. Plasticity rule: $E / a^{c} \rightarrow \alpha k\left(i, N_{j}\right)$, where $c \geq 1, \alpha \in\{+,-, \pm, \mp\}, k \geq 1$, $1 \leq j \leq\left|R_{i}\right|$, and $N_{j} \subseteq\{1, \ldots, m\}$

- syn $\subseteq\{1, \ldots, m\} \times\{1, \ldots, m\}$, with $(i, i) \notin$ syn for $1 \leq i \leq m$, are synapses between neurons;

- in, out $\in\{1, \ldots, m\}$ indicate the input and output neurons, respectively.

Given neuron $\sigma_{i}$ (we can also say neuron $i$ or simply $\sigma_{i}$ if there is no confusion) we denote the set of neuron labels which has $\sigma_{i}$ as their presynaptic neuron as $\operatorname{pres}(i)$, i.e. $\operatorname{pres}(i)=\{j \mid(i, j) \in \operatorname{syn}\}$. Similarly, we denote the set of neuron labels which has $\sigma_{i}$ as their postsynaptic neuron as $\operatorname{pos}(i)=\{j \mid(j, i) \in$ syn $\}$.

Spiking rule semantics in SNPSP systems are similar with SNP systems in 9. However, we do not use forgetting rules and spiking rules with delays in this work. Spiking rules are applied as follows: If neuron $\sigma_{i}$ contains $b$ spikes and $a^{b} \in L(E), b \geq c$, then a rule $E / a^{c} \rightarrow a \in R_{i}$ can be applied. Applying such a rule means consuming $c$ spikes from $\sigma_{i}$, thus only $b-c$ spikes remain in $\sigma_{i}$. Neuron $i$ sends one spike to every neuron in $\operatorname{pres}(i)$. If a rule $E / a^{c} \rightarrow a$ has $E=a^{c}$, we simply write this as $a^{c} \rightarrow a$.

Plasticity rules are applied as follows. If at time $t$ we have that $\sigma_{i}$ has $b \geq c$ spikes and $a^{b} \in L(E)$, a rule $E / a^{c} \rightarrow \alpha k(i, N) \in R_{i}$ can be applied. The set $N$ is a collection of neurons to which $\sigma_{i}$ can connect to (synapse creation) or disconnect from (synapse deletion) using the applied plasticity rule. The rule consumes $c$ spikes and performs one of the following, depending on $\alpha$ :

If $\alpha=+$ and $N-\operatorname{pres}(i)=\emptyset$, or if $\alpha=-$ and $\operatorname{pres}(i)=\emptyset$, then there is nothing more to do, i.e. $c$ spikes are consumed but no synapse is created or removed. For $\alpha=+$ : If $\mid N-$ pres $(i) \mid \leq k$, deterministically create a synapse to every $\sigma_{l}, l \in N_{j}-\operatorname{pres}(i)$. If however $\mid N-$ pres $(i) \mid>k$, then nondeterministically select $k$ neurons in $N$-pres $(i)$, and create one synapse to each selected neuron.

For $\alpha=-$ : If $|\operatorname{pres}(i)| \leq k$, deterministically delete all synapses in pres $(i)$. If however $|\operatorname{pres}(i)|>k$, then nondeterministically select $k$ neurons in pres $(i)$, and delete each synapse to the selected neurons.

If $\alpha \in\{ \pm, \mp\}$ : create (respectively, delete) synapses at time $t$ and then delete (respectively, create) synapses at time $t+1$. Only the priority of application of synapse creation or deletion is changed, but the application is similar to $\alpha \in\{+,-\}$. The neuron is always open from time $t$ until $t+1$, i.e. the neuron can continue receiving spikes. However, the neuron can only apply another rule at time $t+2$.

An important note is that for $\sigma_{i}$ applying a rule with $\alpha \in\{+, \pm, \mp\}$, creating a synapse always involves an embedded sending of one spike when $\sigma_{i}$ connects to a neuron. This single spike is sent at the time the synapse creation is applied. Whenever $\sigma_{i}$ attaches to $\sigma_{j}$ using a synapse during synapse creation, we have $\sigma_{i}$ immediately transferring one spike to $\sigma_{j}$. 
If two rules with regular expressions $E_{1}$ and $E_{2}$ can be applied at the same time, that is, $L\left(E_{1}\right) \cap L\left(E_{2}\right) \neq \emptyset$, then only one of them is nondeterministically chosen and applied. All neurons therefore apply at most one rule in one time step (locally sequential) but all neurons that can apply a rule must do so (globally parallel). Note that the application of rules in neurons are synchronized, that is, a global clock is assumed.

A system state or configuration of an SNPSP system is based on (a) distribution of spikes in neurons, and (b) neuron connections based on the synapse graph syn. We can represent (a) as $\left\langle s_{1}, \ldots, s_{m}\right\rangle$ where $s_{i}, 1 \leq i \leq m$, is the number of spikes contained in $\sigma_{i}$. For (b) we can derive pres $(i)$ and $\operatorname{pos}(i)$ from syn, for a given $\sigma_{i}$. The initial configuration therefore is represented as $\left\langle n_{1}, \ldots\right.$, $\left.n_{m}\right\rangle$, with the possibility of a disconnected graph, i.e. $s y n=\emptyset$. A computation is defined as a sequence of configuration transitions from an initial configuration. A computation halts if the system reaches a halting configuration, that is, a configuration where no rules can be applied and all neurons are open. Whether a computation is halting or not, we associate natural numbers $1 \leq t_{1}<t_{2}<\ldots$. corresponding to the time instances when the neuron out sends a spike out to (or when in receives a spike from) the system.

A result of a computation can be defined in several ways in SNP systems literature, but in this work we use the following as in [9]: we only consider the first two time instances $t_{1}$ and $t_{2}$ that $\sigma_{\text {out }}$ spikes. Their difference, i.e. the number $t_{2}-t_{1}$, is said to be computed by $\Pi$. We denote the set of all numbers computed in this manner by $\Pi$ as $N_{2}(\Pi)$. The subscript indicates that we only consider the time difference between the first two spikes of $\sigma_{\text {out }}$. Also note that 0 cannot be computed as mentioned in section 2 .

In $N_{2}(\Pi)$ the neuron in is ignored, and we refer to this as the generative mode. SNPSP systems in the accepting mode ignore $\sigma_{\text {out }}$ and work as follows: The system begins with an initial configuration, and exactly two spikes are introduced in the system (using $\sigma_{i n}$ ) at times $t_{1}$ and $t_{2}$. The number $t_{2}-t_{1}$ is accepted by $\Pi$ if the computation halts. The set of numbers accepted by $\Pi$ is denoted as $N_{a c c}(\Pi)$. The families of all sets of $N_{\alpha}(\Pi)$, with $\alpha \in\{2, a c c\}$, is denoted as $N_{\alpha} S N P S P$.

\section{An Example of an SNPSP System}

In this section, we provide an example to illustrate the definition and semantics of an SNPSP system. Consider an SNPSP system $\Pi_{e x}$ shown in figure1 1 . Neurons 2 , out $=3, A_{1}$, and $A_{2}$ contain only the rule $a \rightarrow a$ and we omit this from writing. In the initial configuration, at some time $t$, is where only $\sigma_{1}$ has two spikes and $\sigma_{3}$ has only one spike. Neuron 1 is the only neuron with plasticity rules, where $\alpha \in\{+,-\}$, and we have $\operatorname{syn}=\left\{\left(2, A_{1}\right),\left(2, A_{2}\right),\left(A_{1}, 1\right),\left(A_{2}, 1\right)\right\}$.

$\Pi_{e x}$ operates as follows: The application of the two rules in $\sigma_{1}$ are deterministic. Nondeterminism in $\Pi_{e x}$ exists only in selecting which synapses to create to or delete from ${ }^{3}$ Neuron 3 has $n_{3}=1$, so it sends a spike out to the environ-

\footnotetext{
${ }^{3}$ We refer to this later as synapse level nondeterminism
} 


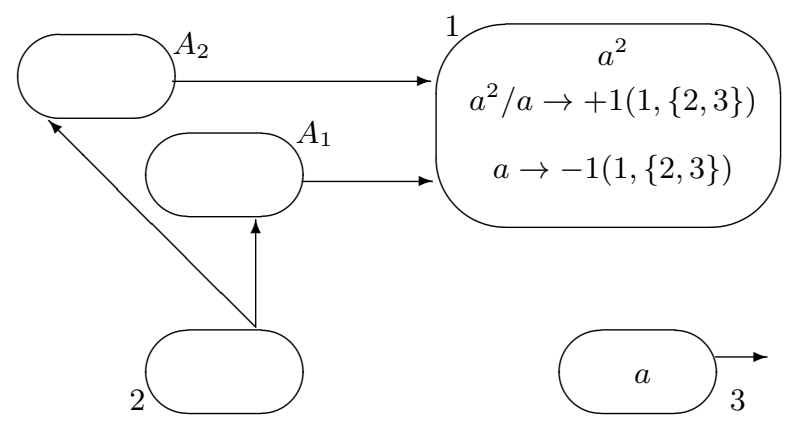

Fig. 1. An SNPSP system $\Pi_{e x}$.

ment, and we label this event as time $t_{1}$. Since $n_{1}=2$, the rule with $\alpha=+$ is applied, so $\sigma_{1}$ nondeterministically selects one (since $k=1$ ) of $\sigma_{2}$ or $\sigma_{3}$ to create a synapse to.

If the synapse $(1,3)$ is created, then $\sigma_{1}$ sends one spike to $\sigma_{3}$ also at time $t_{1}$. The rule with $\alpha=+$ consumes one spike, so $\sigma_{1}$ now has one spike. At time $t_{2}$ the rule with $\alpha=-$ is applied, and synapse $(1,3)$ is deleted. The spike that $\sigma_{3}$ received from $\sigma_{1}$ is sent to the environment at time $t_{2}$ also. Two spikes are sent out to the environment by $\Pi_{e x}$ before it halts. The computed number in this case is then $t_{2}-t_{1}=1$. The computation of $\{1\}$ by $\Pi_{e x}$ is shown in table 1. where (!) means that the output neuron spikes to the environment.

$\begin{array}{ccccccc}\text { time } & \sigma_{1} & \sigma_{2} & \sigma_{3} & \sigma_{A_{1}} & \sigma_{A_{2}} & \text { syn } \\ t & 2 & 0 & 1 & 0 & 0 & \text { syn } \\ t_{1} & 1 & 0 & 1(!) & 0 & 0 & \text { syn } \cup\{(1,3)\} \\ t_{2} & 0 & 0 & 0(!) & 0 & 0 & \text { syn }\end{array}$

Table 1. Computation of $\Pi_{e x}$ computing $\{1\}$.

If however the synapse $(1,2)$ is created, $\sigma_{1}$ sends a spike to $\sigma_{2}$ at time $t_{1}$ during synapse creation. Neuron 2 then sends one spike each to auxiliary neurons $A_{1}$ and $A_{2}$ at time $t_{2}$. Also at time $t_{2}$ is when the rule with $\alpha=-$ is applied, and $(1,2)$ is deleted. $A_{1}$ and $A_{2}$ send one spike each to $\sigma_{1}$, so that $\sigma_{1}$ has two spikes again at time $t_{3}$, as in the initial configuration. As long as $\sigma_{1}$ creates synapse $(1,2)$ instead of $(1,3)$ then $\Pi_{e x}$ keeps receiving two spikes in a loop.

Notice that if at some time $m>1$ the synapse $(1,2)$ is created, it will take $\sigma_{1}$ three time steps for the possibility of creating $(1,3)$ again. Once $(1,3)$ is created, one more step is required for $\sigma_{3}$ to spike to the environment for the second and final time. The computation of $\{4\}$ for example, is shown in table 2 . Therefore, from the operation of $\Pi_{e x}$ we then have $N_{2}\left(\Pi_{e x}\right)=\{1,4,7,10, \ldots\}=$ $\{3 m+1 \mid m \geq 0\}$. Also notice that for $\Pi_{e x}$, its two plasticity rules in $\sigma_{1}$ can be replaced by a single plasticity rule: $a^{2} \rightarrow \pm 1(1,\{2,3\})$. 


$\begin{array}{ccccccc}\text { time } & \sigma_{1} & \sigma_{2} & \sigma_{3} & \sigma_{A_{1}} & \sigma_{A_{2}} & \text { syn } \\ t & 2 & 0 & 1 & 0 & 0 & \text { syn } \\ t_{1} & 1 & 1 & 0(!) & 0 & 0 & \text { syn } \cup\{(1,2)\} \\ t_{2} & 0 & 0 & 0 & 1 & 1 & \text { syn } \\ t_{3} & 2 & 0 & 0 & 0 & 0 & \text { syn } \\ t_{4} & 1 & 0 & 1 & 0 & 0 & \text { syn } \cup\{(1,3)\} \\ t_{5} & 0 & 0 & 0(!) & 0 & 0 & \text { syn }\end{array}$

Table 2. Computation of $\Pi_{e x}$ computing $\{4\}$.

\section{$5 \quad$ Universality of SNPSP Systems}

In this section we show that SNPSP systems are computationally universal, i.e. they characterize $N R E$, both in the accepting and generative modes. In SNPSP systems there are two types of nondeterminism: (1) in selecting which rule to apply in a neuron (rule level nondeterminism), and (2) in selecting which synapses to create to or delete from (synapse level nondeterminism). However, we prove that synapse level nondeterminism is sufficient for universality. Therefore, the SNPSP systems we construct in this work do not involve rule level nondeterminism.

We start with SNPSP systems working in the generative mode, followed by the accepting mode. As in $\Pi_{e x}$ and unless mentioned otherwise, we omit from writing the rules for simple neurons with $a \rightarrow a$ as the only rule. A network of these simple neurons (first referred to in $\underline{\underline{17}}$ ) with rules of a simple form, together with fewer neurons that have plasticity rules, are computationally powerful: they can perform tasks that Turing machines can perform.

\section{SNPSP systems working in the generative mode}

Theorem $1 N R E=N_{2} S N P S P$.

Proof. To prove theorem 1 we only need to prove $N R E \subseteq N_{2} S N P S P$ by simulating a register machine $M$ in generative mode with an SNPSP system. The converse, i.e. $N_{2} S N P S P \subseteq N R E$, is straightforward or the Turing-Church thesis can be invoked. Without loss of generality we may assume for register machine $M=\left(m, I, l_{0}, l_{h}, R\right)$ to have: (a) all registers except register 1 are empty at halting; (b) the output register is never decremented during any computation; and (c) the initial instruction of $M$ is an ADD instruction.

The SNPSP system $\Pi$ simulating $M$ has three modules: the ADD, SUB and FIN modules shown in figures 2, 3, and 4 respectively. All three modules consist of simple neurons and some neurons that have plasticity rules. The ADD and SUB modules simulate the ADD and SUB instructions of $M$, respectively. The FIN module is used to output the computation of $\Pi$ in the generative mode mentioned in section 3 ,

Given a register $r$ of $M$ we have an associated neuron $\sigma_{r}$ in $\Pi$. In any computation, if $r$ stores the value $n$, then $\sigma_{r}$ will contain $2 n$ spikes. For every label 
$l_{i}$ in $M$ we have $\sigma_{l_{i}}$ in $\Pi$. The initial configuration of $\Pi$ is where only $\sigma_{l_{0}}$ has one spike, indicating that instruction $l_{0}$ in $M$ is to be executed. Simulating a rule $l_{i}:\left(\mathrm{OP}(r): l_{j}, l_{k}\right)$ in $M$ means $\sigma_{l_{i}}$ has one spike and is activated. Then, depending on the operation $\mathrm{OP} \in\{\mathrm{ADD}, \mathrm{SUB}\}$, an operation is performed on $\sigma_{r}$. Either $\sigma_{l_{j}}$ or $\sigma_{l_{k}}$ receives a spike and becomes activated. When $M$ executes $l_{h}$ (the label for the halting instruction of $M$ ), $\Pi$ completely simulates the computation of $M$. $\Pi$ then activates $\sigma_{l_{h}}$, and $\sigma_{\text {out }}$ sends two spikes to the environment with a time difference equal to the stored value in register 1.

Module ADD shown in figure 2 simulating $l_{i}:\left(\operatorname{ADD}(r): l_{j}, l_{k}\right)$ : The initial instruction of $M$ is labeled $l_{0}$, and it is an ADD instruction. Assume that instruction $l_{i}:\left(\operatorname{ADD}(r): l_{j}, l_{k}\right)$ is to be simulated at time $t$. Therefore $\sigma_{l_{i}}$ contains one spike while all other neurons are empty except neurons associated with registers. Neuron $l_{i}$ uses its rule $a \rightarrow a$ and sends one spike each to neurons $l_{i}^{1}$ and $l_{i}^{2}$. At time $t+1$ both neurons $l_{i}^{1}$ and $l_{i}^{2}$ send one spike to $\sigma_{r}$ so that $\sigma_{r}$ receives two spikes corresponding to the value one in register $r$ of $M$. Also at time $t+1$ is when neuron $l_{i}^{2}$ sends one spike to $\sigma_{p}$, which is the only neuron in module ADD with plasticity rules.

Once $\sigma_{p}$ receives a spike, $\sigma_{p}$ consumes one spike and at time $t+2, \sigma_{p}$ nondeterministically creates one synapse (since $\alpha= \pm, k=1$ ) to either neuron $l_{j}$ or $l_{k}$. Also at time $t+2, \sigma_{p}$ sends a spike to either neuron $l_{j}$ or $l_{k}$; If synapse $\left(p, l_{j}\right)$ was created then $\sigma_{p}$ removes $\left(p, l_{j}\right)$ afterwards, otherwise $\sigma_{p}$ removes $\left(p, l_{k}\right)$, at time $t+3$.

At time $t+3$ either neuron $l_{j}$ or $l_{k}$ is activated, i.e. receives one spike from $\sigma_{p}$, and can therefore perform the associated instruction. From firing neuron $l_{i}$ the module ADD increments the spikes contained in $\sigma_{r}$ by two and nondeterministically activates either neuron $l_{j}$ or $l_{k}$. We therefore correctly simulate an ADD instruction.

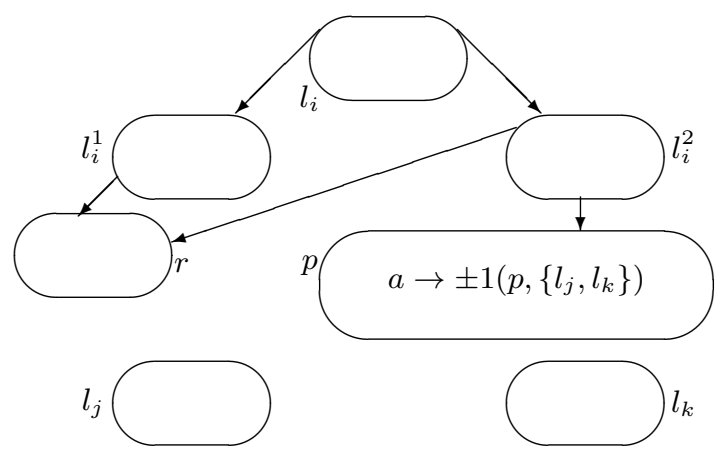

Fig. 2. Module ADD simulating $l_{i}:\left(\operatorname{ADD}(r): l_{j}, l_{k}\right)$.

Module SUB shown in figure 3 simulating $l_{i}:\left(\operatorname{SUB}(r): l_{j}, l_{k}\right)$ : We simulate a SUB instruction with the SUB module as follows: Initially only $\sigma_{l_{i}}$ has one spike and all other neurons are empty except those associated with registers. Let $t$ be 
the time when neuron $l_{i}$ fires, using its rule $a \rightarrow a$ to send one spike to $\sigma_{r}$ and $\sigma_{l_{i}^{1}}$. As with the SUB instruction in $M$, the two cases are when register $r$ stores a nonzero value (execute instruction $l_{j}$ ) or when $r$ stores zero (execute $l_{k}$ ). For the moment let us set $N_{j}=\left\{l_{i}^{2}\right\}$ and $N_{k}=\left\{l_{i}^{3}\right\}$ so that $\left|N_{j}\right|=\left|N_{k}\right|=1$. The case for a general $N_{j}$ and $N_{k}$ will be explained shortly.

If $\sigma_{r}$ contains a nonzero number of spikes at time $t$, then it contains $2 n$ (even numbered) spikes corresponding to $n$ stored in register $r$. At time $t+1$ neuron $r$ has $2 n+1$ (odd numbered) spikes, the additional one spike will come from neuron $l_{i}$ at time $t$. The rule with regular expression $a\left(a^{2}\right)^{+}$is enabled at $t+1$ since $\sigma_{r}$ has an odd number of spikes. At time $t+1$ the rule consumes three spikes and deterministically creates (for the moment) $\left|N_{j}\right|=1$ synapse $\left(r, l_{i}^{2}\right)$, and sends one spike to $\sigma_{l_{i}^{2}}$. At time $t+2$, synapse $\left(r, l_{i}^{2}\right)$ is deleted (since $\alpha= \pm$ ). At time $t+2$ therefore, neuron $l_{i}^{2}$ contains two spikes: one each from neurons $l_{i}^{1}$ and $r$. We then have neuron $l_{i}^{2}$ creating at time $t+2$ a synapse, as well as sending one spike, to neuron $l_{j}$. Neuron $l_{j}$ is therefore activated.

Removing three spikes from $2 n+1$ spikes in $\sigma_{r}$ makes the number of spikes contained in $\sigma_{r}$ even again. In particular, after rule application, $\sigma_{r}$ will contain $2(n-1)$ spikes corresponding to $n-1$ in $r$. Module SUB correctly simulates decreasing the value stored in $r$ by one if $r$ stored a nonzero value, and then executing $l_{j}$.

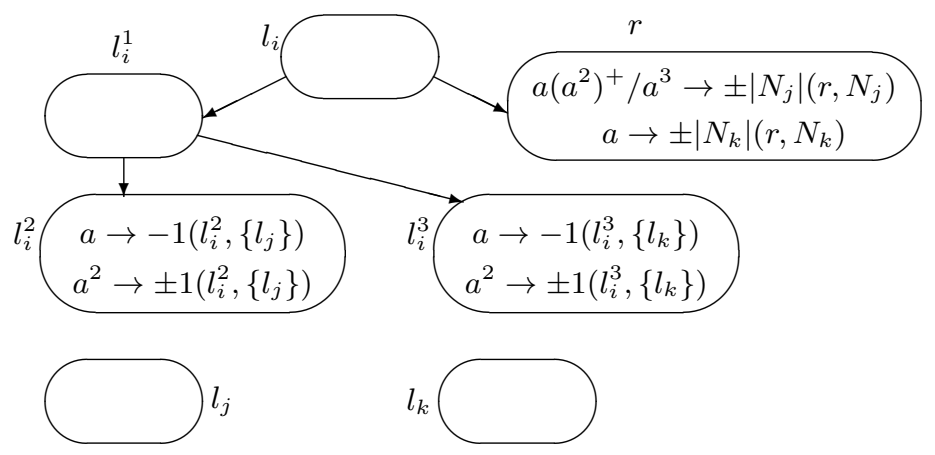

Fig. 3. Module SUB simulating $l_{i}:\left(\operatorname{SUB}(r): l_{j}, l_{k}\right)$.

If $\sigma_{r}$ contains no spikes at time $t$ corresponding to a stored value of zero in $r$, then $\sigma_{r}$ contains one spike at time $t+1$ and applies the rule $a \rightarrow \pm 1\left(r,\left\{l_{i}^{3}\right\}\right)$. This rule consumes one spike, reducing the spikes contained in $\sigma_{r}$ back to zero. The rule also creates synapse $\left(r, l_{i}^{3}\right)$ and sends one spike to neuron $l_{i}^{3}$ at time $t+1$. At $t+2$, synapse $\left(r, l_{i}^{3}\right)$ is deleted. Neuron $l_{i}^{3}$ contains 2 spikes at $t+1$, one each from neurons $r$ and $l_{i}^{1}$. At $t+2$, neuron $l_{i}^{3}$ creates synapse $\left(l_{i}^{3}, l_{k}\right)$ and sends one spike to $\sigma_{l_{k}}$, activating $\sigma_{l_{k}}$. Module SUB correctly simulated maintaining the zero stored in $r$ if $r$ initially stored zero, and then executing $l_{k}$.

For the general case where there are at least two SUB instructions operating on register $r$, this means we have at least two SUB modules operating 
on the same $\sigma_{r}$ : to simulate $l_{x}:\left(\operatorname{SUB}(r): l_{x_{1}}, l_{x_{2}}\right)$ and $l_{y}:\left(\operatorname{SUB}(r): l_{y_{1}}, l_{y_{2}}\right)$, we have to be certain that simulating $l_{x}$ ( $l_{y}$, respectively) only activates either neuron $l_{x_{1}}$ or $l_{x_{2}}\left(l_{y_{1}}\right.$ or $l_{y_{2}}$ respectively). The set $N_{j}\left(N_{k}\right.$, respectively) is defined as $N_{j}=\{j \mid j$ is the second element of the triple in a SUB instruction $\}$ $\left(N_{k}=\{k \mid k\right.$ is the third element of the triple in a SUB instruction $\}$, respectively).

The possibility of at least two SUB modules interfering with each other is removed since each $l_{i}^{2}$ and $l_{i}^{3}, i \in\{x, y\}$, is only activated when a spike from the corresponding $l_{i}^{1}$ is received. Only the specific SUB instruction to be simulated has its corresponding neuron $l_{i}^{2}$ (otherwise $l_{i}^{3}$, depending on the stored value in $r$ ) in a SUB module receive two spikes. The other SUB modules only have their neuron $l_{i}^{2}$ (otherwise, $l_{i}^{3}$ ) receive only one spike. Either neuron $l_{i}^{2}$ or $l_{i}^{3}$ therefore activate the correct neuron: whenever $\alpha= \pm$ (when containing two spikes), or $\alpha=-$ (when containing one spike). If $\alpha=-$ then only synapse removal is performed. Therefore, the SUB instruction is correctly simulated by the SUB module.

Module FIN shown in figure 4 once $l_{h}$ is reached in $M$ : We assume at time $t$ that the computation in $M$ halts, so that instruction with label $l_{h}$ is reached. Also at time $t$ we have $\sigma_{1}$ containing $2 n$ spikes corresponding to the value $n$ stored in register 1 of $M$. Neuron $l_{h}$ sends one spike each to $\sigma_{1}$ and $\sigma_{\text {out }}$. At time $t+1$ neuron out sends the first of two spikes that it will send to the environment before computation halts. Also at time $t+1$ we have $\sigma_{1}$ containing $2 n+1$ spikes. Neuron 1 continuously applies its rule $a^{3}\left(a^{2}\right)^{+} / a^{2} \rightarrow+1(1,\{$ out $\})$ if $\sigma_{1}$ initially contained four or more spikes. The rule performs the following every time it is applied: two spikes are consumed and the synapse (1,out) is deleted ( since $\alpha=-, k=1$ ).

Notice that synapse $(1$, out $)$ does not exist, so the two spikes consumed are simply removed from the system and no synapse is actually removed. Also notice that applying this rule leaves $\sigma_{1}$ with an odd number of spikes afterwards. The value of $k$ in the rule can actually be any positive integer but in this case it is simply set to one. Once the number of spikes in $\sigma_{1}$ is reduced to three, the rule $a^{3} \rightarrow \pm 1(1,\{$ out $\})$ is applied. This rule is applied after $n$ applications of the previous rule. At time $t+n$ the rule removes three spikes and leaves no spikes in $\sigma_{1}$, creates a synapse and sends a spike to $\sigma_{\text {out }}$, and deletes the synapse. Neuron out receives one spike from $\sigma_{1}$ and spikes for the second and final time to the environment at time $t+n+1$. The time difference between the first and second spiking of $\sigma_{\text {out }}$ is $(t+n+1)-(t+1)=n$, exactly the number stored in register 1 of $M$ when the computation of $M$ halted.

From the description of the operations of modules ADD, SUB, and FIN, $\Pi$ clearly simulates the computation of $M$. Therefore we have $N_{2}(\Pi)=N(M)$ and this completes the proof.

\section{SNPSP systems working in the accepting mode}

SNPSP systems in the accepting mode ignore the out neuron and use an in neuron to take in exactly two spikes. The time difference between the two input spikes is the number computed by the system, if the computation halts. Recall 


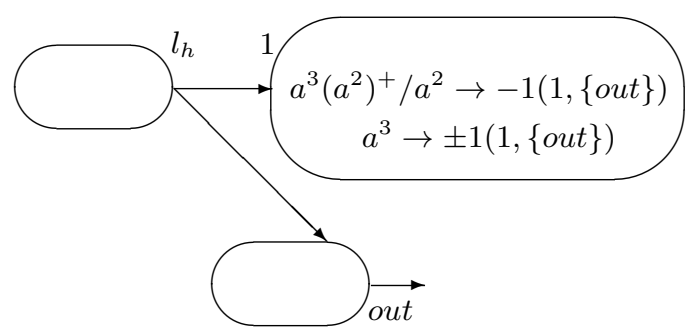

Fig. 4. Module FIN.

that a register machine $M$ is computationally universal even for the deterministic accepting case. The resulting SNPSP system simulating $M$ is therefore simpler compared to the system simulating the generative case as in theorem 1 .

Theorem $2 N R E=N_{a c c} S N P S P$.

Proof. The proof for theorem 2 is a consequence of the proof of theorem 1 Given a deterministic register machine $M=\left(m, I, l_{0}, l_{h}, R\right)$ we construct an SNPSP system $\Pi$ as in the proof of theorem 1 . Since $M$ is deterministic in this case, we modify $\Pi$ so that addition is deterministic and we use an INPUT module instead of a FIN module. As with the proof of theorem 1 the modules will contain simple neurons having only $a \rightarrow a$ as their rule, and some neurons with plasticity rules.

The new module INPUT is shown in figure 5 and functions as follows: All neurons are initially empty and we let the time that the first spike enters the module be $t$. The second and final spike input will arrive at time $t+n$ so that $(t+n)-t=n$ is the value stored in register 1 of $M$. Neuron in uses its rule $a \rightarrow a$ at time $t+1$ to send one spike each to neurons $A_{1}, A_{2}$, and $A_{3}$.

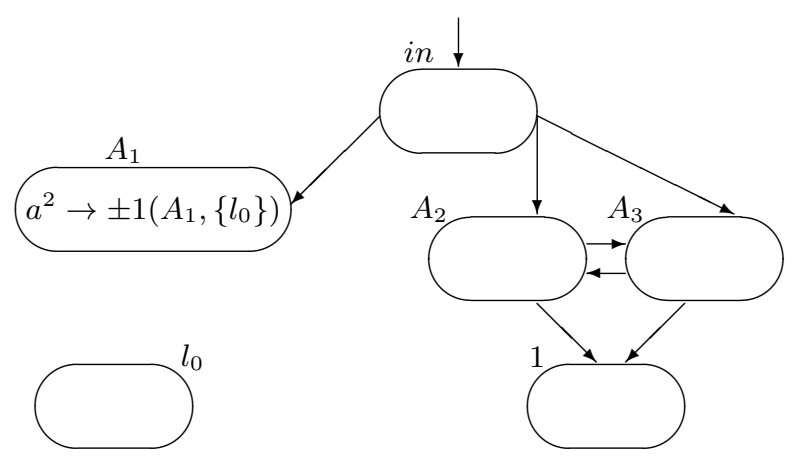

Fig. 5. Module INPUT.

Neurons $A_{2}$ and $A_{3}$ exchange spikes every step from $t+2$ until $t+n+1$. During every exchange, neurons $A_{2}$ and $A_{3}$ increase the spikes inside $\sigma_{1}$ by two. 
Once the second and the final spikes arrive, $\sigma_{i n}$ spikes at $t+n+1$ so that $A_{2}$ and $A_{3}$ now each have two spikes, and therefore cannot apply their only rule $a \rightarrow a$. At time $t+n+1$ neuron $A_{1}$ collects two spikes so it can apply its plasticity rule to activate neuron $l_{0}$. The activation of neuron $l_{0}$ corresponds to the start of the simulation of instruction $l_{0}$ in $M$. Note that from time $t+2$ until $t+n+1, \sigma_{1}$ collects a total of $2 n$ spikes, corresponding to the value $n$ stored in register 1 .

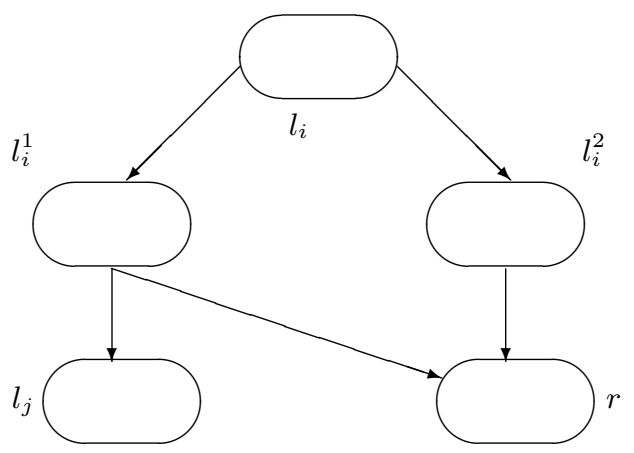

Fig. 6. Module ADD simulating $l_{i}:\left(\operatorname{ADD}(r): l_{j}\right)$.

Since for the accepting case $M$ can be computationally universal even with a deterministic ADD instruction, we have the deterministic ADD module in figure 6. The functioning of the deterministic ADD module is clear and is simpler than the nondeterministic ADD module. The SUB module remains the same as in the proof of theorem 1. Module FIN is not used, while $\sigma_{l_{h}}$ remains in the system except that $\operatorname{pres}\left(\bar{l}_{h}\right)=\emptyset$.

Once $\sigma_{l_{h}}$ receives a spike indicating that the computation of $M$ has halted, the neuron applies its rule $a \rightarrow a$ but does not send its spike to any neuron. Therefore, the computation in $\Pi$ halts only if the computation in $M$ halts, and we have $N_{a c c}(\Pi)=N(M)$ completing the proof.

\section{Spike Saving Mode Universality and Deadlock}

In this section we consider a modification of how plasticity rules are applied: if there is nothing more to do in terms of synapse creation or deletion, we do not consume a spike. We prove that given this modification, SNPSP systems are still universal. Additionally, an interesting property, the deadlock, can occur.

Let us consider again the definition and semantics of plasticity rules in section 3. If $\sigma_{i}$ contains $b$ spikes and a rule $E / a^{c} \rightarrow \alpha k:\left(i, N_{j}\right)$, if $a^{b} \in L(E)$, then the plasticity rule can be applied. Applying the rule means consuming $c$ spikes, and then performing plasticity operations depending on the value of $\alpha$ and $k$. If however $\operatorname{pres}(i)=\emptyset$, there is nothing more to do for $\alpha=-$ : This is because there is no synapse to delete, since $\sigma_{i}$ is not a presynaptic neuron of any other 
neuron. Such a case is seen with the module FIN in figure 4. Note that $c$ spikes are still consumed, even if we later realize that there is nothing more to do. Such a case also exists for $\alpha=+$.

An interesting modification of this plasticity rule mode of operation is as follows: during the "nothing more to do" case, i.e. whenever $\alpha \in\{+,-\}$ and $N_{j}-\operatorname{pres}(i)=\emptyset$ or $\operatorname{pres}(i)=\emptyset$, we save spikes so that they are not consumed. As an analogy, if we pay a certain money or currency to perform a task, then this saving mode means our money is returned if we discover that the task has been performed already. In contrast, the nonsaving mode in section 3 is analogous to paying money to perform a task, regardless if the task is actually performed or not.

A natural inquiry from modifying SNPSP systems from nonsaving to saving mode is: are SNPSP systems in the saving mode still universal? The answer is affirmative, and requires a modification of the FIN module in figure 4 . The modification is the basis for the next result. We denote by $S N P S P_{s}$ the family of sets computed by an SNPSP system in saving mode.

Theorem $3 N R E=N_{2} S N P S P_{s}$

Proof. The SUB and FIN modules make use of the nonsaving mode in theorem 1 . We only need to modify the two modules, while the ADD module remains intact. We denote the modified SUB and FIN modules as $\mathrm{SUB}^{\prime}$ and $\mathrm{FIN}^{\prime}$, respectively. Module FIN $^{\prime}$ is shown in figure 7. For $\mathrm{FIN}^{\prime}$, we add one new neuron $\sigma_{p}$ and a new synapse $(1, p)$. Neuron $p$ does not have any rules and $\operatorname{pres}(p)=\emptyset$. We also remove the plasticity rule $a^{3}\left(a^{2}\right)^{+} / a^{2} \rightarrow-1(1,\{$ out $\})$ and replace it with a spiking rule $a^{3}\left(a^{2}\right)^{+} / a^{2} \rightarrow a$.

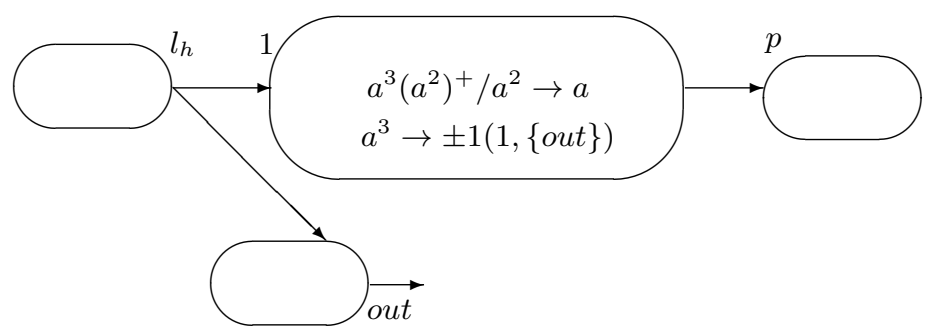

Fig. 7. Module FIN'.

Note that we still do not use forgetting rules. The purpose of the new rule in $\sigma_{1}$ is to reduce the number of spikes each time step by 2 in $\sigma_{1}$ until only 3 remain. Once there are 3 spikes in $\sigma_{1}$, then the remaining rule $a^{3} \rightarrow \pm 1(1,\{$ out $\})$ is applied. The new $\sigma_{p}$ functions as a trap or repository for the spikes removed from $\sigma_{1}$. We then have FIN $^{\prime}$ functioning as it should be: delaying the second spike of $\sigma_{\text {out }}$ for $n$ steps, as required, and as was performed by FIN also.

\footnotetext{
${ }^{4}$ Or in the case of neurons, the quanta of energy which is the spike.
} 


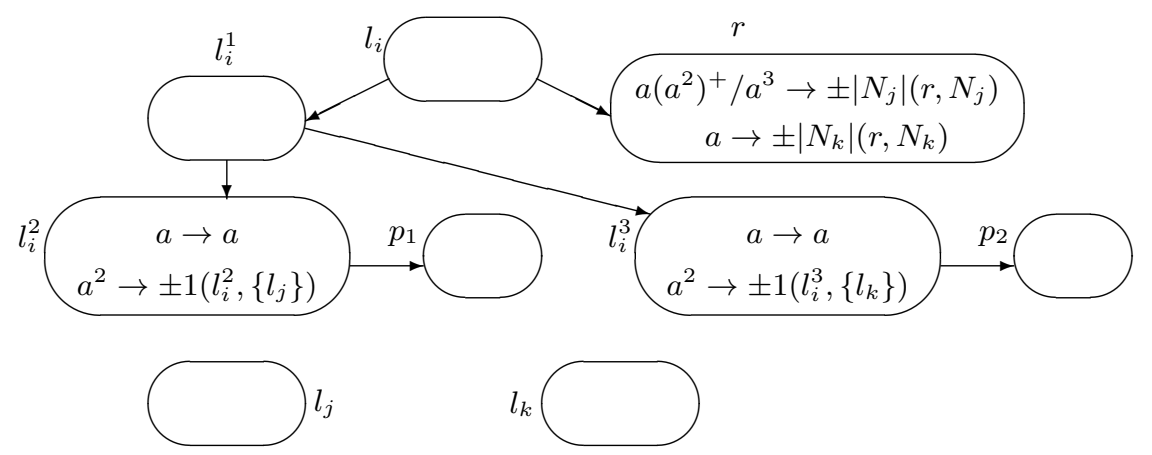

Fig. 8. Module $\mathrm{SUB}^{\prime}$ simulating $l_{i}:\left(\operatorname{SUB}(r): l_{j}, l_{k}\right)$.

Module $\mathrm{SUB}^{\prime}$ is shown in figure 8 . For $\mathrm{SUB}^{\prime}$, we add trap neurons $\sigma_{p_{1}}$ and $\sigma_{p_{2}}$ for every neuron $l_{i}^{2}$ and $l_{i}^{3}$ in a SUB module, respectively. We also add for each SUB module, the synapses $\left(l_{i}^{2}, p_{1}\right)$ and $\left(l_{i}^{3}, p_{2}\right)$. We then replace the rule $a \rightarrow-1\left(l_{i}^{2},\left\{l_{j}\right\}\right)$ in neuron $l_{i}^{2}$ (rule $a \rightarrow-1\left(l_{i}^{3},\left\{l_{k}\right\}\right)$ in neuron $l_{i}^{3}$, respectively) with a spiking rule $a \rightarrow a$. Similar to the trap neuron in FIN $^{\prime}$, the trap neurons in $\mathrm{SUB}^{\prime}$ receive the unwanted spikes from neuron $l_{i}^{2}$ (or $l_{i}^{3}$ ) so that interference does not occur. Therefore, $\mathrm{SUB}^{\prime}$ functions similarly to SUB, and as required.

Corollary 1 NRE $=N_{a c c} S N P S P_{s}$

Corollary 1 follows from theorem 3 , since in the accepting mode from theorem 2, the saving or nonsaving mode is of no consequence. An interesting property that arises when using the saving mode is the existence of a deadlock. During the saving mode, deadlock occurs when a plasticity rule is applied because the regular expression is satisfied, but no actual work is performed by the neuron. The result is a state where no further computation can continue, and the system is stuck or trapped in the current configuration, even if a rule can always be applied. More formally, a deadlock state (or configuration) occurs if a $\sigma_{i}$ can apply at least one plasticity rule and we have the following sequence of events during the same time step: $a^{c}$ spikes are consumed, however the applied plasticity rule either has $\alpha=+$ and $N-\operatorname{pres}(i)=\emptyset$, or $\alpha=-$ and $\operatorname{pres}(i)=\emptyset$; since there is nothing more to do in such a case, $a^{c}$ spikes are returned to $\sigma_{i}$.

Lemma 1 A deadlock can exist in an arbitrary SNPSP system in saving mode having at least two neurons, with $\alpha \in\{+,-\}$.

Proof. Let us first consider when $\alpha=+$, and we define a $\Pi_{(+)}$as follows.

$$
\Pi_{(+)}=\left(\{a\}, \sigma_{i}, \sigma_{j}, \operatorname{syn}_{(+)}\right)
$$

where $\sigma_{i}=\left(1, R_{i}\right), R_{i}=\{a \rightarrow+1(i,\{j\})\}, \sigma_{j}=(0, \emptyset)$, and $\operatorname{syn}_{(+)}=\{(i, j)\}$. Figure 9 provides an illustration for $\Pi_{(+)}$. We have that $\Pi_{(+)}$has exactly one 
synapse between $\sigma_{i}$ and $\sigma_{j}$, and exactly one rule (a plasticity rule) in $\sigma_{i}$. Once the plasticity rule is applied, the saving mode requires us to consume one spike first. The plasticity rule has $\alpha=+$ and $k=1=|\operatorname{pres}(i)|$. Therefore we must create a new synapse from $\sigma_{i}$ to $\sigma_{j}$. However, the synapse $(i, j)$ already exists and we return the consumed spike, as dictated by the saving mode. Therefore $\Pi_{(+)}$is stuck in the current and only configuration: the regular expression of the only rule is satisfied, however, we cannot perform further computations.

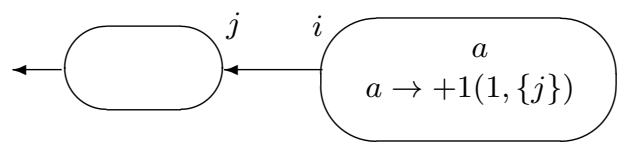

Fig. 9. The SNPSP system $\Pi_{(+)}$with a deadlock from the proof of lemma 1

Now let us consider a similar SNPSP system $\Pi_{(-)}$defined as follows.

$$
\Pi_{(-)}=\left(\{a\}, \sigma_{i}, \sigma_{j}, \operatorname{syn}_{(-)}\right)
$$

where $\sigma_{i}=\left(1, R_{i}\right), R_{i}=\{a \rightarrow-1(i,\{j\})\}, \sigma_{j}=(0, \emptyset)$, and $\operatorname{syn}_{(-)}=\emptyset$. In a similar way, the one and only rule of $\Pi_{(-)}$is always applied, since the regular expression is satisfied. However, there is no synapse between $\sigma_{i}$ and $\sigma_{j}$, since $\operatorname{syn}_{(-)}=\operatorname{pres}(i)=\emptyset$, so there is nothing to do. The saving mode dictates that the consumed spike must be returned and the system remains in a deadlock.

An important problem (e.g. see [10]) is whether it is decidable to have an arbitrary SNPSP system in saving mode arrives at a state of deadlock or not. Not surprisingly, the answer to this problem is a negative one, as given by the next result.

Theorem 4 It is undecidable whether an arbitrary SNPSP system $\Pi^{\prime}$ in saving mode, with at least two neurons, reaches a deadlock.

Proof. We consider an arbitrary recursively enumerable set of natural numbers which we denote $D$. We can have a register machine, an SNP system, or an SNPSP system generate the elements of $D$. For simplicity, we then choose to construct an SNPSP system $\Pi$ similar to section 5 such that $D=N_{2}(\Pi)$. The system $\Pi$ produces two spikes (using its output neuron) if and only if $D$ is non-empty, and this task is undecidable. Furthermore, we construct an SNPSP system $\Pi^{\prime}$ using $\Pi$ as a submodule. We refer to figure 10 for a graphical representation for $\Pi^{\prime}$ instead of a formal definition.

If $D$ is nonempty (this means $\sigma_{\text {out }}$ of $\Pi$ spikes twice), then $\sigma_{i}$ receives two spikes and it can apply its rule. However, from lemma 1 we know that the application of the rule in $\sigma_{i}$ results in a state of deadlock. The problem of realizing if $D$ is nonempty is undecidable. Therefore, a deadlock is reached if and only if the set $D$ is nonempty, which happens to be undecidable. 


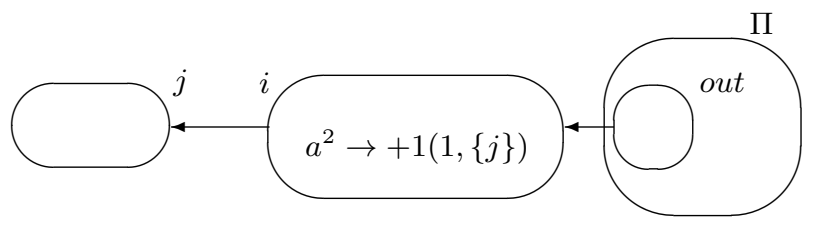

Fig. 10. The SNPSP system $\Pi^{\prime}$ for the proof in theorem 4 .

\section{Solving Subset Sum}

In this section we provide a solution to the numerical NP-complete problem Subset sum. The solution provided here is nondeterministic and semi-uniform. By uniform solution we mean that we construct only one solution (in this case, an SNPSP system) for any instance of the problem. The construction of a semiuniform solution depends on specific instances of a problem, and semi-uniform if the construction is done in polynomial time. We refer to [11] 12] 16] 23] for details of uniformity in solutions.

In 11 for example, the Subset sum problem was also solved in a semi-uniform way using SNP systems with extended rules. Additionally, their solution used modules that have neurons operating in deterministic or nondeterministic ways, and applying rules in sequential or maximally parallel manner. A follow-up and improved solution was then given in [12. For the solution we present here, unless otherwise mentioned, the SNPSP systems work in nonsaving mode as in section 3. The problem Subset sum can be defined as follows:

Problem: Subset Sum.

- Instance: $S$, and a (multi)set $V=\left\{v_{1}, v_{2}, \ldots, v_{n}\right\}$, with $S, v_{i} \in \mathbb{N}$ and $1 \leq$ $i \leq n$

- Question: Is there a $\operatorname{sub}\left(\right.$ multi)set $B \subseteq V$ such that $\sum_{b \in B} b=S$ ?

We can now have the following last result.

Theorem 5 There exists a semi-uniform SNPSP system solving any instance $W=\left(S, V=\left\{v_{1}, \ldots, v_{n}\right\}\right)$ of Subset Sum, with the following parameters:

- synapse level nondeterminism only,

- without forgetting rules and rules with delays,

- computes in constant time.

Proof. First, we formally describe the SNPSP system $\Pi_{\mathrm{ss}}(W)$ that solves instance $W$ of the problem.

$\Pi_{\mathrm{ss}}=\left(\{a\}, \sigma_{1}, \ldots, \sigma_{n}, \sigma_{1^{(1)}}, \ldots, \sigma_{1^{\left(2 v_{1}\right)}}, \ldots, \sigma_{n^{(1)}}, \ldots, \sigma_{n^{\left(2 v_{n}\right)}}, \sigma_{\text {out }}\right.$, syn, out $)$ where:

(1) For $1 \leq i \leq n, \sigma_{i}=\left(v_{i}, R_{i}\right)$, with $R_{i}=\left\{a^{v_{i}} \rightarrow+v_{i}\left(v_{i},\left\{i^{(1)}, \ldots, i^{\left(2 v_{i}\right)}\right\}\right)\right\}$; 
(2) For $1 \leq j \leq v_{n}, \sigma_{i(j)}=(0,\{a \rightarrow a\})$;

(3) For $v_{n}+1 \leq k \leq 2 v_{n}, \sigma_{i(k)}=(0, \emptyset)$;

and

$-\sigma_{\text {out }}=\left(0,\left\{a^{S} \rightarrow a\right\}\right)$

- syn $=\left\{\left(1^{(1)}\right.\right.$, out $), \ldots,\left(1^{\left(v_{n}\right)}\right.$, out $), \ldots,\left(n^{(1)}\right.$, out $), \ldots,\left(n^{\left(v_{n}\right)}\right.$, out $\left.)\right\} ;$

We refer to figure 11 for a graphical representation of $\Pi_{s s}$. In figure 11, for simple neurons that only have the rule $a \rightarrow a$, we omit the writing of the rule as in section 5. Note however that in the definition of $\Pi_{s s}$, some neurons actually do not have rules in them. The distinction between these empty neurons and the simple neurons will be explained shortly. Next, we provide the functioning of $\Pi_{\mathrm{ss}}$, which we divide into three stages for clarity:

Stage 1: This stage consists of operations performed by neurons $\sigma_{1}$ to $\sigma_{n}$, where $\sigma_{i}$ initially contains $v_{i}$ spikes. These are the neurons from (1). Each $\sigma_{i}$ consumes all the initial $v_{i}$ spikes, to create $v_{i}$ number of synapses. Notice that each $\sigma_{i}$ has $\alpha=+$ and $k=v_{i}$, but can nondeterministically select among $2 v_{i}$ number of neurons to create a synapse to. Once every $\sigma_{i}$ has finished selecting and creating synapses to $v_{i}$ number of neurons, we move to the next stage.
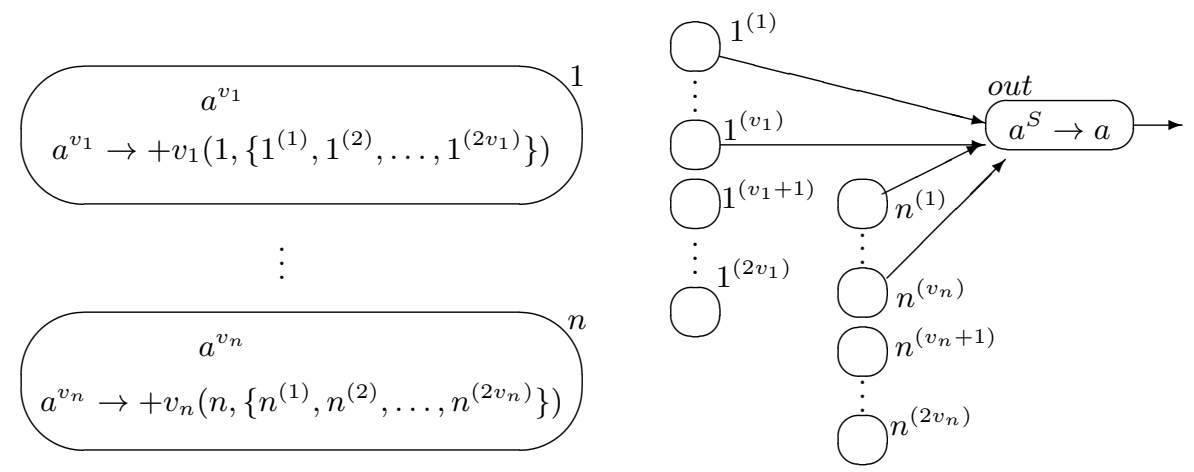

Fig. 11. The SNPSP system $\Pi_{\text {ss }}$ solving Subset Sum.

Stage 2: This stage consists of operations performed by neurons $\sigma_{1^{(1)}}$ up to $\sigma_{1^{\left(2 v_{1}\right)}}$ associated with $\sigma_{1}$ (from stage 1 ), up to the neurons $\sigma_{n^{(1)}}$ up to $\sigma_{n^{\left(2 v_{n}\right)}}$ associated with $\sigma_{n}$ (from stage 1 ). These are the neurons from $(\mathbf{2})$ and $(\mathbf{3})$. Note in the definition that given the $2 v_{i}$ neurons in this stage associated with $\sigma_{i}$ in stage 1 , the first half of these neurons (i.e. $\sigma_{i(1)}$ up to $\left.\sigma_{i^{\left(v_{i}\right)}}\right)$ are simple neurons with the rule $a \rightarrow a$. The second half (i.e. $\sigma_{i^{\left(v_{i}+1\right)}}$ up to $\sigma_{i^{\left(2 v_{i}\right)}}$ ) are empty neurons, without initial spikes or rules. Also note that neurons in the first half each have a synapse to $\sigma_{\text {out }}$, while the second half do not have synapses, i.e. they are all traps, where each of their presynaptic sets is empty. 
Since the nondeterminism in stage 1 is synapse level, in some computations some (or all) simple neurons will each receive a spike, while in other computations some (or all) empty neurons each receive a spike. For the computations that send a spike to the empty neurons, these neurons function as trap or repository neurons. Consequently, the spikes in these trap neurons can also be removed using forgetting or plasticity rules. The computations that have some (or all) simple neurons in this stage then send one spike each to $\sigma_{\text {out }}$, leading us to the next stage.

Stage 3: This final stage checks the existence of an $S$ number of spikes from stage 2. If there are $S$ spikes in $\sigma_{\text {out }}$ this means the answer to this instance of the Subset Sum is affirmative, and one spike is sent to the environment. This spike, affirming the answer to the problem instance, is sent out to the environment 3 time steps since stage 1 . If however the spikes sent to $\sigma_{\text {out }}$ from stage 2 do not add up to $S$, this means the answer to the problem instance is negative. Therefore, at time step 3 no spike is sent to the environment.

\section{Final Remarks}

Before we provide the final discussion, we briefly recall the contributions of this work: Section 3 introduced the structural plasticity feature in the SNP systems framework, providing a partial answer to an open problem in [22] about dynamism only for synapses. The "programming" of the system is dependent on the way neurons (dis)connect to each other using synapses.

In section 5 and 6 we proved that SNPSP systems in the generative and accepting cases are universal, for both the saving and nonsaving modes. The universality results hold even if we impose the following restrictions on SNPSP systems: only 5 among the 17 total neurons for all modules 5 use plasticity rules, only synapse level nondeterminism exist, neurons without plasticity rules are simple (having only the rule $a \rightarrow a$ ), and without using forgetting rules or delays. Additionally, a state known as deadlock can arise during saving mode. Reaching such a state for an arbitrary SNPSP system in saving mode is undecidable. In section 7 we provided a nondeterministic and semi-uniform solution to Subset Sum, computing in 3 time steps.

The deadlock state does not seem to exist in nonsaving mode. Does this mean that saving mode is "better" than nonsaving mode? Both modes have the same expressiveness, but perhaps the (non)existence of a deadlock is useful for more practical purposes, e.g. modeling or analysis of systems. For example, reaching a deadlock can be interpreted as reaching an unwanted state or fault in a network or system. This is an interesting theoretical and practical question. Deadlocks and other state types, as applied to modeling and analysis, have an extensive body of research in many graphical formalisms such as Petri nets [10]. Several works have related Petri nets, SNP systems, and other P systems. See

${ }^{5} \mathrm{ADD}, \mathrm{SUB}$, and FIN module neurons in the saving and generative case, since the accepting case requires a lesser number of neurons with plasticity rules. 
for example $\underline{3}$ and references therein. A state of undecidable deadlock can also occur in systems in [20.

The solution provided in section 7 is a preliminary one: it is of course interesting to ask other ways to encode instances of other NP-complete problems for (non)deterministic synapse selection. What about (non)deterministic, uniform solutions? In computer science we work to have efficient solutions, seeking minimal parameters. However, and as we mentioned in section 1 biology is usually not space efficient: there are billions of cells in the human body, and billions of neurons each with thousands of synapses in our brains. So the solution in section 7 may not immediately appeal to our search for minimal parameters, but it seems biologically appealing. Depending on the instance to be solved, the number of neurons for example (and possibly the spikes and synapses) in $\Pi_{s s}$ can be exponential with respect to the instance size.

Other parameter modifications of computational interest abound: what about parallel synapse creation-deletion? The semantics in section 3 for $\alpha \in\{ \pm, \mp\}$ is sequential, requiring 2 time steps to perform such rules with such values for $\alpha$. We only list here a few other complexity parameters that can be used: the size of syn during a computation, e.g. the number of synapses created (deleted), or a bound on the size of syn (related to synaptic homeostasis); in the universality proofs we had $\alpha \in\{+,-, \pm\}$, so can we have universality with $\alpha$ having at most 2 values only? Normal forms for SNPSP systems are interesting as well.

SNPSP systems and the proofs in section 5 are reminiscent of the more generalized extended SNP systems and their universality proofs in 1. Investigating the relation of ESNP and SNPSP systems seems interesting. More recently, SNP systems with rules on synapses were shown to be universal [25]. In these systems, neurons are only spike repositories, and the spike processing (including nondeterminism) are done in the synapses. Investigating theoretical or practical usefulness of such systems, together with structural plasticty, is also interesting.

Lastly, SNPSP systems can be equipped with one input and one output neurons, providing us with a transducer. Such transducers can be used to compute (in)finite strings as in [21. Furthermore, SNPSP systems with multiple inputs or outputs are also interesting, for computing strings or vectors of numbers as in $\underline{1}$ again.

\section{Acknowledgements}

F.G.C. Cabarle is supported by a scholarship from the DOST-ERDT Philippines. T. Song is supported by the China Postdoctoral Science Foundation Project (No. 2014M550389). H.N. Adorna is funded by a DOST-ERDT research grant and the Semirara Mining Corporation professorial chair of the College of Engineering, UP Diliman. M.J. Pérez-Jiménez acknowledges the support of the Project TIN201237434 of the "Ministerio de Economía y Competitividad" of Spain, co-financed by FEDER funds. The authors are also grateful to three anonymous referees for their useful comments. 


\section{References}

1. Alhazov, A., Freund, R., Oswald, M., Slavkovik, M.: Extended Spiking Neural P Systems. H.J. Hoogeboom et al. (Eds.): WMC 7, LNCS 4361, pp. 123-134 (2006)

2. Butz, M., Wörgötter, F., van Ooyen, A.: Activity-dependent structural plasticity. Brain Research Reviews 60, pp. 287-305 (2009)

3. Cabarle, F.G.C., Adorna, H..: On Structures and Behaviors of Spiking Neural P Systems and Petri Nets. E. Csuhaj-Varjú et al. (Eds.): CMC 2012, LNCS 7762, pp. 145-160 (2013)

4. Cabarle, F.G.C, Adorna, H., Ibo, N.: Spiking neural P systems with structural plasticity. ACMC2013, Chengdu, China, 4-7 November (2013)

5. Cavaliere, M., Ibarra, O., Păun, G., Egecioglu, O., Ionescu, M., Woodworth, S.: Asynchronous spiking neural P systems. Theoretical Computer Science, 410, pp. 2352-2364 (2009)

6. García-Amau, M., Pérez, D., Rodríguez-Patón, A., Sosík, P.: Spiking Neural P Systems: Stronger Normal Forms. International journal of unconventional computing, vol 5(5), pp. 411-425 (2009)

7. Gutiérrez-Naranjo, M.A., Pérez-Jiménez, M.J.: Hebbian Learning from Spiking Neural P Systems View. D. Corne et al. (Eds.) WMC9, LNCS 5391, pp. 217-230 (2009)

8. Ibarra, O., Păun, A., Păun, G., Rodríguez-Patón, A., Sosík, P., Woodworth, S.: Normal forms for spiking neural P systems. Theoretical Computer Science vol 372(23) pp. 196-217 (2007)

9. Ionescu, M., Păun, Gh., Yokomori, T.: Spiking Neural P Systems. Fundamenta Informaticae, vol. 71(2,3), pp. 279-308 (2006)

10. Iordache, M., Antsaklis, P.: Deadlock and Liveness Properties of Petri Nets. Supervisory Control of Concurrent Systems: A Petri Net Structural Approach. pp. 125-151. Birkhäuser Boston (2006)

11. Leporati, A., Zandron, C., Ferretti, C., Mauri, G.: Solving Numerical NP-Complete Problems with Spiking Neural P Systems. Eleftherakis et al. (Eds.): WMC8 2007, LNCS 4860, pp. 336-352 (2007)

12. Leporati, G., Mauri, G., Zandron, C., Păun, G., Pérez-Jiménez, M.: Uniform solutions to SAT and Subset Sum by spiking neural P systems. Natural Computing vol. 8, pp. 681-702 (2009)

13. Minsky, M.: Computation: Finite and infinite machines. Englewood Cliffs, NJ: Prentice Hall, (1967)

14. Pan, L., Păun, G.: Spiking neural P systems with anti-spikes. J. of Computers, Communication, \& Control. vol IV(3), pp. 273-282 (2009)

15. Pan, L., Păun, G.: Spiking Neural P Systems: An improved normal form. Theoretical Computer Science vol 411(6), pp. 906-918 (2010)

16. Pan, L., Păun, Gh., Pérez-Jiménez, M.J.: Spiking neural P systems with neuron division and budding. Science China Information Sciences. vol. 54(8) pp. 1596-1607 (2011)

17. Pan, L., Wang, J., Hoogeboom, J.H.: Spiking Neural P Systems with Astrocytes. Neural Computation 24, pp. 805-825 (2012)

18. Păun, Gh.: Computing with membranes. Journal of Computer and System Science, vol. 61(1), pp. 108-143 (1999)

19. Păun, Gh.: Membrane Computing: An Introduction. Springer (2002)

20. Păun, Gh.: Spiking Neural P Systems with Astrocyte-like Control. J. Universal Computer Science. vol. 13(11), pp. 1707-1721 (2007) 
21. Păun, Gh., Pérez-Jiménez, M.J., Rozenberg, G.: Computing Morphisms by Spiking Neural P Systems. International Journal of Foundations of Computer Science. vol. 8(6) pp. 1371-1382 (2007)

22. Păun, Gh., Pérez-Jiménez, M.J.: Spiking Neural P Systems. Recent Results, Research Topics. A. Condon et al. (eds.), Algorithmic Bioprocesses, Springer (2009)

23. Păun, Gh., Rozenberg, G., Salomaa, A. (Eds) The Oxford Handbook of Membrane Computing, OUP (2010)

24. Song, T., Pan, L., Păun, G.: Asynchronous spiking neural P systems with local synchronization. Information Sciences, 219, pp.197-207 (2013)

25. Song, T., Pan, L., Păun, G.: Spiking neural P systems with rules on synapses. Theoretical Computer Science vol. 529(10) pp. 82-95 (2014)

26. Turing, A. Intelligent Machinery. in Essential Turing: Seminal Writings in Computing, Logic, Philosophy, Artificial Intelligence, and Artificial Life: Plus The Secrets of Enigma. Copeland, B. (Ed.). OUP (2004)

27. Wang, J., Hoogeboom, H.J., Pan, L.: Spiking Neural P Systems with Neuron Division. M. Gheorghe et al. (Eds.): CMC 2010, LNCS 6501, pp. 361-376 (2010) 